\section{OC-064 HEPATIC FIBROSIS IN PEOPLE WITH TYPE 2 DIABETES MELLITUS: THE EDINBURGH TYPE 2 DIABETES STUDY}

doi:10.1136/gutjnl-2012-302514a.64

1J R Morling,* ${ }^{2} \mathrm{M}$ W Strachan, ${ }^{2} \mathrm{R}$ M Williamson, 'J F Price, ${ }^{3} \mathrm{~J}$ A Fallowfield, ${ }^{4}$ I N Guha. ${ }^{1}$ Centre for Population Health Sciences, University of Edinburgh, Edinburgh, UK; ${ }^{2}$ Metabolic Unit, Western General Hospital, Edinburgh, UK; ${ }^{3}$ MRC Centre for Inflammation Research, University of Edinbugh, Edinburgh, UK; ${ }^{4}$ Nottingham Digestive Disease Centre BRU, University of Nottingham, Nottingham, UK

Introduction People with type 2 diabetes mellitus (T2DM) have a higher prevalence of and higher mortality rates from liver-associated disease than the non-diabetic population of equivalent age. Early identification of liver disease followed by appropriate surveillance and intervention should be beneficial to the expanding population of older people with T2DM. We aimed to determine the prevalence and progression of hepatic fibrosis in a representative sample of older adults with T2DM

Methods 1066 participants of the Edinburgh Type 2 Diabetes Study, a large, randomly-selected population of patients with T2DM aged 60-75 years were invited to assessment on two occasions. At baseline, the Enhanced Liver Fibrosis Panel (ELF) was measured and demographics and diabetes history were recorded. ELF was repeated approximately 3 years later. The presence of fibrosis was determined as: none/mild $<7.7$, moderate $7.7-<9.8$, severe $\geq 9.8$. Fibrosis progression was investigated for association with demographics, diabetes history and alcohol intake using logistic regression.

Results Average follow-up was 2.7 years. At baseline $(n=530), 3.1 \%$ subjects had no/mild fibrosis, $85.1 \%$ moderate fibrosis and $11.9 \%$ severe fibrosis. Follow-up prevalences $(n=806)$ were $1.9 \%, 79.3 \%$, and $18.9 \%$ respectively. 489 subjects had both baseline and followup ELF results. 61 (12.5\%) patients progressed to the most severe fibrosis stage between baseline and follow-up. None of the factors investigated were independently associated with progression to severe fibrosis: age, sex, social deprivation, diabetes-related factors (fasting glucose, HbA1c, diabetes duration, treatment type or location) or alcohol use.

Conclusion These preliminary data suggest that considerable hepatic fibrosis is present in older patients with T2DM. ELF is well validated for diagnosing severe hepatic fibrosis and it is of concern that in $<3$ years, almost $13 \%$ of patients progressed to this level. Further work is planned to determine factors affecting progression and to develop prognostic models for the development of hepatic fibrosis in people with T2DM.

Competing interests None declared.

\section{OC-065 TARGETED INHIBITION OF TISSUE FACTOR AND THROMBIN ON CD31 EXPRESSING CELLS SUPPRESSES HEPATIC FIBROSIS IN CCL4 TREATED MICE}

doi:10.1136/gutjnl-2012-302514a.65

${ }^{1}$ G Petts, ${ }^{*}{ }^{2} \mathrm{~A}$ Dhar, ${ }^{1} \mathrm{H}$ Kudo, ${ }^{2} \mathrm{~F}$ Sadiq, ${ }^{3} \mathrm{O}$ Anstee, ${ }^{2} \mathrm{Y}$ Kallis, ${ }^{4} \mathrm{~A}$ Dorling, ${ }^{1} \mathrm{R}$ Goldin, ${ }^{2} \mathrm{M}$ Thursz. ${ }^{1}$ Centre for Pathology, Imperial College, London, UK; ${ }^{2}$ Department of Medicine, Imperial College, London, UK; ${ }^{3}$ Institute of Cellular Medicine, Newcastle University, Newcastle-upon-Tyne, UK; ${ }^{4}$ MRC Centre for Transplantation, King's College, London, UK

Introduction Recent evidence suggests a role for the coagulation cascade in promoting liver fibrosis. However, the cellular basis for this relationship is unclear. In order to explore this relationship we employed two unique transgenic mice strains expressing membrane-tethered tissue factor pathway inhibitor (TFPI) or hirudin (antithrombin) fusion proteins driven by a CD31 promoter. These strains allow for the selective inhibition of tissue factor (TF) or thrombin on endothelial cells, monocytes and platelets expressing CD31.
Aims To evaluate the impact of the targeted inhibition of tissue factor and thrombin on effector cells of liver fibrosis in murine hepatic fibrosis induced by $\mathrm{CCl}_{4}$.

Methods Liver fibrosis was induced in CD31-TFPI, CD31-Hirudin and wild type control mice with 4 weeks carbon tetrachloride $\left(\mathrm{CCl}_{4}\right)$ administered by intraperitoneal injection. Animals were culled and livers extracted for histological and biochemical analysis. Fibrosis was scored using a four point semi-quantitative system and quantified by digital image analysis to determine percentage area of fibrosis. Immunohistochemistry to determine $\alpha$-SMA expression, a marker of hepatic stellate cell activation was performed and the mean number of activated stellate cells was quantified per high power field.

Results The percentage area of fibrosis was significantly less in CD31-TFPI $(1.89 \% \pm 0.26, \mathrm{p}=0.001)$ and CD31-Hirudin mice $(1.04 \% \pm 0.16, p=0.00003)$ in comparison to control mice $(3.66 \% \pm$ 0.39 ). Semiquantitative fibrosis scoring showed a significant difference between the CD31-TFPI (median 2/4) and CD31-Hirudin (median 3/4) mice in comparison with control mice (median $3 / 4$ $\mathrm{p}=0.009$ ) but the difference between the two transgenic strains was not significant. Both transgenic strains demonstrated a significantly reduced mean number of $\alpha$-SMA stellate cells per high power field in comparison to control mice (CD31-TFPI vs control, 7.4 vs 29.4, $\mathrm{p}=0.0002$; CD31-hirudin vs control, 5.25 vs 29.4, $\mathrm{p}=0.0002$ ).

Conclusion CD31 targeted inhibition of TF and thrombin significantly reduces liver fibrosis and stellate cell activation in a murine $\mathrm{CCl}_{4}$ model. The data supports the use of this novel murine model as a tool for investigating the cellular biology of the role of coagulation in liver fibrogenesis. It will also provide information for developing new treatments of human liver fibrosis.

Competing interests None declared.

\section{OC-066 TOLL LIKE RECEPTOR 4 ANTAGONIST PREVENTS ACETAMINOPHEN INDUCED ACUTE LIVER FAILURE IN MICE: A NOVEL THERAPEUTIC STRATEGY}

doi:10.1136/gutjnl-2012-302514a.66

${ }^{1} \mathrm{~N}$ Shah, ${ }^{2} \mathrm{D}$ Dhar, ${ }^{1} \mathrm{M}$ Jover-Cobos, ${ }^{1} \mathrm{~N}$ A Davies, ${ }^{1} \mathrm{R} \mathrm{P}$ Mookerjee, ${ }^{1} \mathrm{R}$ Jalan. ${ }^{1}$ Department of Hepatology, UCL Institute of Hepatology, London, UK; ' ${ }^{2}$ Department of Surgery, UCL Institute of Hepatology, London, UK

Introduction Without transplantation, about $40 \%$ of patients with acute liver failure (ALF) die. Its treatment is an unmet need Unregulated inflammation plays an important role in the pathogenesis. Our hypothesis is that Toll like receptor 4 (TLR 4) is critical in the progression of inflammation in ALF. The aims of the study were to determine whether (1) administration of a novel TLR4 antagonist to an acetaminophen (APAP) model of ALF in mice would prevent liver injury. (2) TLR4 antagonist in ALF prolongs survival. (3) TLR4 KO are protected from the liver injury induced by APAP.

Methods Study 1: 3 groups of CD1 mice were studied ( $\mathrm{n}=6$ in each group) Naive, APAP, $500 \mathrm{mg} / \mathrm{kg}$ single dose IP after overnight fasting). APAP+TLR4 antagonist, STM28 (Osaka, Japan); $20 \mu \mathrm{g}$ IP, $1 \mathrm{~h}$ prior to administration of APAP and $6 \mathrm{~h}$ late). Study 2: 3 groups of $\mathrm{C} 57 \mathrm{BL} / 6$ mice were studied $(\mathrm{n}=6)$ in each group. Naive, APAP $(500 \mathrm{mg} / \mathrm{kg}$ single dose IP), APAP+TLR4 antagonist; IAXO (Innaxon) $3 \mathrm{mg} / \mathrm{kg}$ IP, $1 \mathrm{~h}$ prior to administration of APAP and $6 \mathrm{~h}$ later. Study 3: C57BL/6 TLR4 KO were administered APAP $500 \mathrm{mg} /$ $\mathrm{kg}$ and the respective naive controls were administered $500 \mathrm{mg} / \mathrm{kg}$ of saline. Biochemistry was measured using COBAS integra, Cytokines in plasma and tissue homogenate of liver, kidney and brain were measured using ELISA bead array. Brain water was measured using the dry-wet weight method. 
Results Both the TLR4 antagonist's (STM28 and IAXO compound) reduced the plasma liver enzymes, ammonia and creatinine to the control level. The increase in the plasma TNF- $\alpha$ induced by APAP $(45 \pm 3.2)$ was attenuated following TLR4 antagonist (20 \pm 2.3$)$ $(p<0.01)$. This was associated with a reduction in brain water $(p<0.01)$. Both the TLR4 antagonists significantly reduced pericentral necrosis of the liver. Both these interventions showed an improvement in the survival as using the log rank test $(p<0.02)$. TLR4 KO mice treated with APAP were protected from liver necrosis and had significantly better survival than wild type controls $(\mathrm{p}<0.002)$.

Conclusion These data provides evidence for an important of TLR4 in APAP induced ALF and provide the rationale for a clinical trial of this strategy in ALF.

Competing interests None declared.

\section{OC-067 THE BALANCE BETWEEN T HELPER 17 AND FOXP3+ T REGULATORY CELLS IN PATIENTS WITH CHRONIC HEPATITIS C: RELATION TO DISEASE ACTIVITY AND HEPATIC FIBROSIS}

doi:10.1136/gutjnl-2012-302514a.67

${ }^{1} \mathrm{H}$ A El Aggan, ${ }^{*} \mathrm{~N}$ Farahat, ${ }^{3} \mathrm{~L}$ Younis, ${ }^{1} \mathrm{~A}$ ElYamany, ${ }^{1} \mathrm{D}$ Mostafa. ${ }^{1}$ Department of Medicine, Hepatobilary Unit; ${ }^{2}$ Department of Clinical Pathology; ${ }^{3}$ Department of Pathology, Faculty of medicine, Alexandria, Egypt

Introduction $\mathrm{T}$ helper (Th)17 cells, a newly identified subset of Th cells, are major mediators of inflammation-associated disease and have a reciprocal developmental relationship with the immunosuppressive $\mathrm{T}$ regulatory (Treg) cells, which actively restrain the inflammatory response. The present work was designed to study the balance between Th17 cells and Treg cells in patients with chronic hepatitis $\mathrm{C}(\mathrm{CHC})$ in relation to disease activity and severity of hepatic fibrosis.

Methods Twenty patients with treatment-naive $\mathrm{CHC}$ and 20 healthy subjects were included in the study. The Th cells, Th17 cells and Treg cell subsets in fresh whole blood samples were identified as $\mathrm{CD}^{+} \mathrm{CD}^{+}, \mathrm{CD}^{+} \mathrm{IL}_{17 \mathrm{~A}^{+}}$and $\mathrm{CD} 4^{+} \mathrm{CD} 25^{+} \mathrm{FoxP}^{+}$cells respectively using flow cytometry and expressed as percentages of total lymphocytic count. Serum IL17 levels were measured using solid phase sandwich enzyme linked immunosorbant assay kit. Liver biopsies from patients with CHC were examined to assess histological activity grade and fibrosis stage according to METAVIR scoring system. Liver-infiltrating $\mathrm{CD}^{+}$(Th cells), IL17A ${ }^{+}$cells (Th17 cells) and FoxP3 ${ }^{+}$cells (Treg cells) were detected by immunohistochemical staining and their proportions were determined as ratios of infiltrating $\mathrm{CD}^{+}$Th cells.

Results Patients with CHC showed significant increases in the percentage of Th17 cells, Th17 cells/FoxP3 ${ }^{+}$Treg cells ratio in peripheral blood and serum IL17 levels and a significant decrease in the percentage of circulating FoxP3 ${ }^{+}$Treg cells compared with healthy subjects $(p<0.01)$. The percentages of peripheral blood $\mathrm{CD}^{+}$Th cells were not statistically different between the two groups $(p=0.284)$. The proportions of liver-infiltrating IL17A ${ }^{+}$cells and $\mathrm{FoxP}^{+}$cells of the total intrahepatic $\mathrm{CD}^{+}{ }^{+}$cell population were inversely correlated and showed positive correlations with the percentages of circulating Th17 cells and FoxP3 ${ }^{+}$Treg cells respectively in patients with CHC $(p<0.05)$. The METAVIR necroinflammation grade and fibrosis stage [but not serum HCV RNA levels] were directly correlated with the proportion of intrahepatic IL17A $^{+}$cells and IL17A ${ }^{+}$cells/FoxP3 ${ }^{+}$cells ratio and serum IL17 levels and were inversely correlated with the proportion of liverinfiltrating FoxP3 ${ }^{+}$cells $(\mathrm{p}<0.05)$.

Conclusion In patients with $\mathrm{CHC}$, the $\mathrm{CD} 4^{+}$Th cell phenotype is skewed towards the IL17 producing-Th phenotype. The imbalance between Th17 and Foxp3 ${ }^{+}$Treg cells plays an important role in disease progression and hepatic fibrosis in $\mathrm{CHC}$.

Competing interests None declared.

\section{OC-068 ALBUMIN IS CENTRAL IN MEDIATING THE CARDIO- RENAL DYSFUNCTION OF CIRRHOSIS: A STUDY IN ANALBUMINAEMIC RATS}

doi:10.1136/gutjnl-2012-302514a.68

R Garcia Martinez, ${ }^{*}$ A Habtesion, G Tritto, M Jover, R Jalan, N Davies. Department of Medicine, UCL Hepatology, London, UK

Introduction Albumin is a multifunctional protein which is reduced in concentration and function in liver disease. Albumin infusion prevents and improves renal dysfunction in patients with advanced liver failure but the mechanisms of its beneficial properties are unclear. We hypothesised that albumin is central in the maintenance of cardio-renal function in cirrhosis and albumin impairment worsens outcome. In order to answer this question we investigated analbuminaemic rats, characterised by lack of albumin but with normal protein concentration, following induction of cirrhosis with bile duct ligation (BDL).

Methods Male Sprague-Dawley (SD) and Nagase analbuminaemic (NAR) rats were studied 6 weeks after BDL or sham surgery $(n=6$ sham-SD, 8 sham-NAR, 7 SD-BDL, 7 NAR-BDL). Rats underwent systemic mean arterial pressure (MAP) and portal pressure (PP) assessment under terminal anaesthesia. Plasma and urine were collected for measurements of renal function and protein profile. Plasma renin activity (PRA) was measured as a marker of cardiorenal dysfunction. Urinary neopterin, a marker of macrophage activation was assessed.

Results NARs showed plasma total protein concentration similar to SD despite lack of albumin before $(72 \pm 15$ vs $82 \pm 8)$ and after BDL (67 \pm 7 vs $75 \pm 22$ ). After BDL both groups of animals showed histological evidence of severe liver damage, though the NARs showed a significantly worse systemic haemodynamics with lower MAP ( $p=0.01)$, evidence of renal dysfunction indicated by higher plasma creatinine and higher PRA $(\mathrm{p}<0.05)$ compared with SD (Abstract OC-068 figure 1). There was a significant inflammatory response following BDL in both groups showed by an increase in urinary neopterin which was found to correlate with PRA ( $r=0.59$, $\mathrm{p}<0.01)$

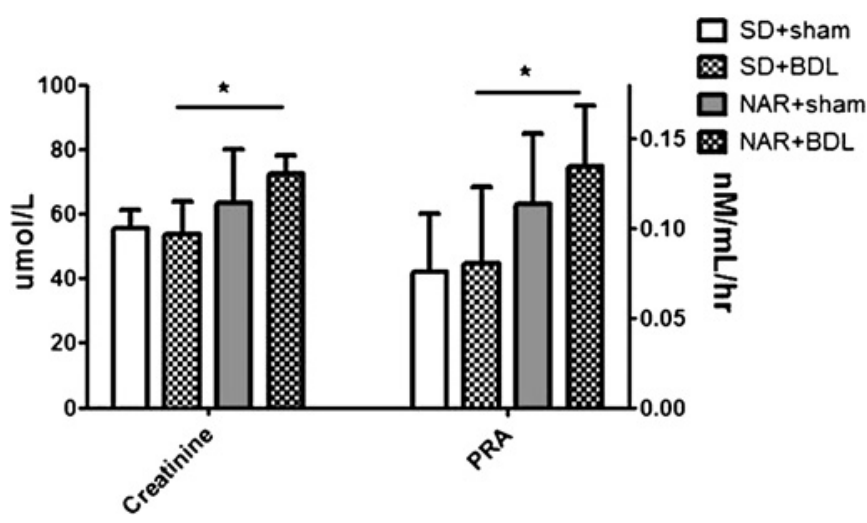

Abstract OC-068 Figure 1 Plasma creatinine concentration and plasma renin activity in the different groups of animals

Conclusion A lack of albumin was associated with a raised PRA and a marked deterioration in systemic haemodynamics and renal function after liver injury (BDL), despite normal total plasma protein concentration. This worsened outcome in the absence of albumin strongly supports the central role of albumin in 